\title{
Qualitative Open Science - Pain Points and Perspectives
}

\author{
Sarahanne M. Field, Don van Ravenzwaaij, Merle-Marie Pittelkow, \\ Joyce M. Hoek, \& Maarten Derksen \\ Rijksuniversiteit Groningen
}

Word count: 6660

Corresponding author: Sarahanne M. Field

University of Groningen, Department of Psychology

E-mail should be sent to s.m.field@rug.nl

\begin{abstract}
Adopting some practical elements of open science - a movement whose goal is to make scientific research available for everyone - presents unique challenges for qualitative researchers, particularly when it comes to data sharing. In this article, we discuss the issue of open qualitative data, arguing that while concerns about ethics and loss of data quality are legitimate, they do not pose so great a problem as to preclude qualitative researchers from effectively practicing open science. We describe the cost-benefit balance that each qualitative researcher takes into account as they choose whether or not to share their data, and highlight the fact that qualitative research practice lends itself to transparency and integrity by its reliance on reflexivity, and other practices such as member checking and using multiple coders. We conclude with a reminder to readers that fruitful open science does not require for one to engage in all possible practices for a given study; only those which are appropriate and feasible.
\end{abstract}

Keywords: Open science, qualitative methods, reflexivity 


\section{Introduction}

Research is getting a global makeover thanks to open science (henceforth, OS). Defined by Vicente-Saez and Martinez-Fuentes as "transparent and accessible knowledge that is shared and developed through collaborative networks", OS has been called a "disruptive phenomenon' (p. 428; Vicente-Saez \& Martinez-Fuentes, 2018), reforming how scientific inquiry is conducted. Although the OS movement emerges from a troubling context (so the popular narrative goes, see e.g., Spellman, Gilbert, \& Corker, 2018; McBee, Makel, Peters, \& Matthews, 2018; Sarafoglou, Hoogeveen, Matzke, \& Wagenmakers, 2020), it is gaining momentum on its own as a new approach to science.

\section{Open Science: What is it and what can it achieve?}

OS can be described as a philosophy, in terms of how people engage with it practically, or in terms of its purported benefits. In abstract terms, OS can be described as science that is practiced with transparency and integrity, and with an emphasis on collaboration and inclusion. It "encompasses a range of behaviours that aim to include the transparency of scientific research and how widely it is communicated" (p. 1; Robson et al., n.d.). OpenAIRE (www.openaire.eu) describes OS as the opening up of the 'research lifestyle', from the conception of research ideas, to the creation of materials and collection of data, to publication and re-use of research findings. The various practices of OS generally come with the promise of many benefits for the scientific community too: they facilitate sharing and scrutiny of research materials, and can further collaboration.

OS promises a range of advantages to society at large, as well as to academia and individual actors working within it. It can play a role in accelerating discovery, and advancing trustworthy and replicable research. OS can facilitate the development and advancement of science (Standen, 2019), and to further the valorization of scientific knowledge and development of policies. Citizen science, which refers to the involvement of non-scientists, in the scientific process can improve science on the societal level. Engagement with science can help lay-people enrich their scientific literacy, and be an opportunity to make genuinely important and impactful contributions to research. Engaging society with the scientific process means that the needs and interests of society are represented faithfully.

Crucially, opening up the scientific process and sharing research output has the potential to help make science more inclusive. Although issues with inclusion and equality in science are far-reaching and systematic (and thus impossible to combat easily) "inclusive knowledge infrastructures enable diverse agents to participate and collaborate in research processes", say Pownall and colleagues (p. 12; 2021). Open access can help this process along, as it provides access to papers to researchers at institutions which do not have subscriptions to large publishers, thus facilitating the involvement of researchers which have historically been marginalized. For instance, researchers living in the global South may be better able to engage in scientific discourse. Open access also allows laypeople to consume research output which is relevant to them and their families.

OS practices which involve open code, open data and open access, whereby researchers make their study materials, data and publications freely available, benefit the academic community, because they maximize the use of resources (like funding), which tend to be scarce. 
On the level of individual actors, opening the peer review process possibly leads to higher-quality and more constructive reviews (Kowalczuk, Dudbridge, Nanda, Harriman, \& Moylan, 2013; Walsh, Rooney, Appleby, \& Wilkinson, 2000), and allows researchers to get credit for a traditionally thankless and arduous, yet necessary academic task. Individual engagement in OS practices like using the registered report submission format, benefits researchers in that they receive peer review on study plans before data are collected, and 'inprinciple acceptance' which all but guarantees publication even in the face of null or messy results. The researcher's discipline also benefits from better-planned research methodology, and, likely, higher quality research output (see e.g., Field et al., 2020).

\section{An Emphasis on Replication in Open Science}

The OS movement has given rise to countless new practices, tools and organizations. Understandably, many of these initiatives focus on remedying problems originally recognized in disciplines where discourse about the crisis of confidence - a crisis fueled to a large extent by concerns about replication - first began to gain momentum. Increased transparency is seen as crucial in improving the reproducibility of results. Pratt, Kaplan and Whittington (2020) neatly describe the logic: "to be trustworthy, research must be replicable; and to better ensure replicability, researchers need to be more transparent about their data and methods." (p. 2). Replication, appropriate use of statistics, sharing data and code, and methods of reducing researchers' bias and degrees of freedom are, understandably, popular topics under discussion in the OS community.

Although we do not agree with many of the assertions Pratt and colleagues make in their 2020 article (such as that the OS movement insists on exact replication as proof of trustworthiness, or those about preregistration and data sharing; pages 5, 6 and 8), we do agree that shackling or associating replication with trustworthiness is problematic for qualitative research. To explicate, for the purposes of the current narrative: linking scientific legitimacy (in terms of, for instance, transparency and validity), however indirectly or implicitly, with OS practices (where replication and reproducibility are seen as essential) carries the risk of excluding qualitative researchers from the OS discussion.

Moreover, it can have the effect of diminishing the value of qualitative research as, by these standards, it is not classified as a legitimate science. Equally importantly, it may discourage the development of qualitative OS practices. Lorenz and Holland (2020) write that when qualitative research is pegged as unscientific due to its lack of reliance on scientific concepts like replication, people are neglecting two important things. First, replication aims to reduce error in estimating a true effect, and second, qualitative researchers achieve the aim of rigor through other means (like maintaining detailed metadata and using multiple coders).

In this article we investigate the compatibility of the various OS practices with qualitative research. We discuss the OS practices which are perhaps most complex to apply in qualitative research settings, outlining the arguments presented in the literature, and supplementing these with our own perspectives. We describe a 'cost-benefit balance' that the qualitative researcher must keep in mind as they consider opening their data, and follow with suggestions for how to circumvent some of the costs associated with practicing open qualitative research, with specific applicability for the social sciences. We conclude with a consideration of how OS can be seen as a buffet, with qualitative researchers taking 
what they need and sharing their own expertise with quantitative scientists in how to bring integrity and validation to scientific research through their own approaches.

\section{Qualitative Research}

The tradition of qualitative research is defined largely by its primary goal: to understand the experiences of humans, and their reflections on those experiences. The methods employed by qualitative researchers in reaching this goal are used to produce richly textured, detailed, non-numerical data (Jackson, Drummond, \& Camara, 2007). Qualitative methods tend to rely heavily on the use of the researcher as an instrument (Lincoln \& Guba, 1985), which means the role of the researcher is highly personal, as they seek to understand - rather than to just explain - social and behavioral phenomena (Stake, 1995). Qualitative researchers rarely intentionally manipulate participant variables, such as is the case with much quantitative empirical research, rather they observe the actions of participants or encourage them to talk, and interpret the resulting observations and discourse within a theoretical framework.

The methodological choices of the qualitative researcher reflect the need to produce high-quality, high-definition evidence (though there is much overlap in these methods, and some inconsistency in terminology; Vaismoradi, Turunen, \& Bondas, 2013). As it is with quantitative research, the approach to data collection (e.g., interviews, case study, participant observation), analysis (e.g., thematic, discourse or narrative) and interpretation (structured with the help of a paradigm or theoretical framework) chosen by the researcher depends on what issues are to be explored.

For instance, if one seeks to identify prominent themes in community discourse, one might conduct a series of interviews and conduct a thematic analysis on the resulting interview transcripts by identifying patterns in the text. If, instead, the researcher's interest is to explore the culture of a community (to understand its rules of conduct and conditions for membership, for instance), one might conduct ethnography. This entails fieldwork, where the researcher immerses themselves in a community with the goal of observing their behavior from close up.

One important common thread among these and other qualitative approaches to research, is the emphasis on the close relationship between the researcher, the participant and the data they co-produce during the research process. Data are not collected, but rather produced by researcher and participants together. An interview, for example, is a collaborative effort in which the researcher's presence (physical, or even online) and their contribution to the conversation are essential. No amount of standardization of the questions and procedure can erase the role of the interviewer. Similarly, interpreting the data is not a process that can be formalized in a strict procedure. It is always, to some extent, a creative act of the interpreter.

\section{Open Science - Qualitative Application}

The OS movement has spawned a great number of initiatives. The shared goal of these initiatives is to increase transparency, which is thought to improve the quality and integrity of the scientific process in turn. Many of them are readily applicable to all traditions of scientific inquiry, including qualitative research disciplines. For instance, open access 
publishing and open peer review pertain to any kinds of science which use journal publishing as their primary means of disseminating findings. Qualitative researchers should, on the whole, find few obstacles to engaging in these. Other practices such as data sharing are less easily applicable, and may 'look' different when used for more qualitative research traditions. Controversy surrounding the applicability of data sharing practices to qualitative research methods has led to a series of thought-provoking publications, which we use in this paper to support and further our own arguments.

\section{Open Qualitative Data}

Open data refers to data and metadata which have been shared. More specifically, it refers to the process by which this sharing occurs. Open data may mean that others may use the data themselves, for their own research purposes, or use it to verify existing findings. Although OS is not equated to sharing data, for many OS practitioners, data sharing is a crucial part of the general philosophy of transparent scientific practice, and thus very important for the OS movement.

Barriers to Sharing. A great deal of articles in the literature raise serious issues regarding opening qualitative data, with two prominent issues dominating the discourse. First, legitimate concerns have been voiced over sharing rich participant data, because these data often contain identifying information (Corti, 2000; Pratt et al., 2020). Although some qualitative data sets (like those of interview studies, for instance) can be anonymized, the internet and the ways it can be used makes it almost impossible to guarantee complete anonymity to participants. Anonymization (whether it is totally effective or not) is not possible for many cases (such as, for example, those involving ethnography, or case-studies).

However, for some, qualitative data sets repositories which are specifically designed for use with sensitive data might offer a tangible option. Mannheimer, Pienta, Kirilova, Elman, and Wutich (2019) provide a great exploration of the possibilities of storing and sharing these kinds of data. They discuss how institutional libraries can play a key role in helping researchers engage in transparency while still protecting their participants, and respecting the trust they place in researchers as they share their stories and experiences. They provide information about ways of modifying data to anonymize it without it losing its value, and discuss how sharing of sensitive data may be made legally and ethically feasible through the use of data use agreements which set out how data can be shared, with whom, and under what special conditions.

With improving institutional support and infrastructure, sharing qualitative data may become feasible in more cases than it currently is. Manneheimer and colleagues emphasize that although improved institutional support does not offer a panacea for the data sharing challenges faced by qualitative researchers, they can "act as advisors and sounding boards for examining these continuing challenges, and they can connect researchers with other relevant specialists to discuss potential solutions." (p. 648)

Another valuable resource which describes how qualitative data and materials were shared in a large team-science context is the article by Borgman, Wofford, Golshan and Darch (2021). They share what they learned in a project spanning 20 years about continuity over generations of researchers, transfer of data between various software tools and research methods, and discuss the role professional data managers played in the project. 
Jones et al. (2018) formally describe different levels of processing and data sharing, and different access scenarios for these levels for qualitative data. These levels of processing, already defined in earth science by NASA's Earth Observation System Data and Information System range from level 0 to level 4, where 0 refers to completely unprocessed, raw data, and level 4 to model outputs or analytical results like derived variables. Other dimensions to the level of processing exist, including the presence of metadata and other associated variables. The tables featured in the Jones article illustrate the variety of options which are available to suit different cases of qualitative data sharing, and through them the authors hope to encourage qualitative researchers to open their data in whatever way is most appropriate.

A useful counterpoint to keep in mind too, is that while issues frequently center on participants' wishes to stay anonymous, some may instead welcome the association of their identities with their perspectives. As Miller (2015) and Pain (2012) explain, many participants are willing and even eager to receive attribution for their contributions to qualitative research.

A second concern raised frequently in the literature is that if (non-sensitive or sufficiently anonymized) qualitative data were shared, estranged from its collector and custodian, it would be missing the context and richness it accumulates during its collection (e.g., see Corti, 2000), and present "significant epistemological challenges" for the use of open qualitative data (p. 3; Chauvette, Schick-Makaroff, \& Molzahn, 2019). This loss of background can constitute a loss of the value of the data, according to some (e.g., Pratt et al., 2020), and at least gives the 're-users' of the data problems with interpretability and validity. Chauvette, Schick-Makaroff and Molzahn (2019) share concerns that the use of others' fieldwork documentation might lead to misinterpretation and misunderstanding of phenomena. They raise the point that field notes are often written in a researcher's own style of shorthand, reflecting feelings and observations that are unique to that researcher, especially in the case of ethnography. They also point out that not all observations lend themselves to notes: nonverbal communication between individuals, for instance, and not all context-dependent knowledge is even noted down at all.

As we mentioned before, qualitative data are always produced by researcher and participants together. Qualitative data are not only 'theory-laden', as quantitative data are, but also 'researcher-laden'. Several authors, however, have suggested that this need not hinder the use of other researchers' data, as long as their contribution to the data is made visible. Corti (2000), for instance, suggests that good documentation removes the need to have 'been there' during data collection. That is, researchers can share much information which provides context and richness to the data, such as study design, interview protocols, and coding strategies, as well as reflections and observations collected at the same time as the data themselves (Dienlin et al., 2021). While Corti concedes that even the best documentation does not replace the tacit knowledge gained by the data collector, a tradition of sharing such accompanying documentation already exists among qualitative research teams, dismantling the argument that you cannot meaningfully interpret findings if you were not there for the data collection.

Original researcher notes may be hard to parse for fellow scientists, in part because they are highly personalized (Borgman et al., 2021), but perhaps researchers can adapt their note-taking for future potential re-use, and can work closely with potential data reusers as collaborators. If a researcher works with a plan to share their data, they are likely 
to be able to supplement field notes with extra details and information that might assist other researchers' comprehension. The original researcher's note-taking style could adapt to be less cryptic, and the method of processing field notes after they have been taken might include emphasis on making sure they are intelligible for others, for example, which might be helpful in cases where researchers are working as a team, or when their findings have to be shared with others like co-authors or supervisors. In the case of interviews, for instance, one can provide an introduction to the interview itself, including a description of the setting, the ambiance, and some nonverbal communication which took place in the interview, rather than just transcribing the interview audio. Some qualitative researchers already complement their data with such details, however, if the practice capturing of granular data and metadata were the norm, it would help overcome some of the challenges of sharing qualitative research material. Moreover, when qualitative data are shared, the new interpretation could be checked with the original researcher who collected the data, analogous to member checking.

Hesitation to share data is not only related to ethical and methodological integrity. As Lezaun and Montgomery (2015) remind us, people are reluctant to share what is most valuable to them. In the context of research, data and metadata are valuable. Most qualitative research products are the result of years of establishing rapport with communities and individuals, and time spent establishing inside knowledge of the people under study. Data, for qualitative research are made up of, among other elements, field notes and transcripts. These tend to take time and effort to generate, even for shorter studies with a small amount of data. It is hard to give away know-how such as this, especially when the reward for doing so is abstract, seems distant, and the work for it often goes uncredited.

Benefits to Sharing. Though sharing qualitative data is a pain point for many qualitative researchers, the benefits of sharing cannot be underestimated. Sharing fosters transparency and innovation, and leads to "encouraging diversity in analysis and opinion" (p. 2; Chauvette et al., 2019). Sharing data can lead to a more efficient and economical use of those data (Corti \& Fielding, 2016), and can assist other researchers in assessing new theories and validating existing findings (Coltart, Henwood, \& Shirani, 2013). All of these advantages can engender robust scientific dialogue, say Chauvette and colleagues.

Importantly, sharing qualitative data can also confer benefits to participants. For instance, consider research fatigue - the burden placed on individuals and communities who are research participants. Sharing data can mitigate this issue say Jones and et al. (2018), particularly for participants who are or have been the subject of repeated data collection, this benefit is likely quite tangible and meaningful. Jones and colleagues share the case of four indigenous communities in the Mackenzie Delta region of the Canadian Arctic, which became the most studied communities in the country during the 1960s and 1970s, following a surge in interest in natural resources found in the area.

Additionally, say Jones and colleagues, sharing data benefits participants in that researchers can use existing data to improve the appropriateness and utility of research questions and projects as they can be refined and refocused as they are revisited by other researchers. 


\section{Open Materials}

Sharing any kind of code which is used in research allows others to verify that a study's findings are valid, and to potentially reuse stimulus sets, analyses or plots in other studies, or on other data. Although qualitative research does not (typically) involve statistical analyses and data plots, some kinds of qualitative analysis rely heavily on codes of another kind, which also facilitate data analysis. We suggest that code sharing is a practice which qualitative researchers can engage in, albeit in a form somewhat different to that which is typically referred to as open code. This is especially important to consider if data are shared.

In qualitative research, 'coding' refers to the process of identifying sections of text or portions of other data (such as photos or video), and identifying relationships and classifying important themes in them to organize and interpret within the research context. Qualitative researchers who conduct coding typically develop a codebook. This is a list of codes with associated definitions, examples and descriptions. A codebook also describes certain exclusionary criteria, i.e., how codes should not be defined. The codebook includes codes which are defined either before the data are analyzed, in the case of deductive coding processes, or codes which have been identified by the data, in the case of inductive coding. Other kinds of protocol-related information can be shared in the same vein as code too, such as interview protocols, and the plans for analysis strategies, for instance.

Roberts, Dowell and Nie (2019) provide a comprehensive discussion about the use of codebooks in qualitative research, in relation to their use, testing and sharing. They discuss how a well-described qualitative methods section, including detailed and reflexive accounts of methods and procedures, helps establish a clear 'trail of evidence' for the validity of research. While reports of many qualitative studies which make use of techniques like thematic analysis do not share useful and detailed descriptions of codebook development, these would undoubtedly allow for validation of qualitative findings in many cases, and even reproduction, if that is the goal (Roberts et al., 2019). Codebooks can be shared alongside data and other documentation (like field notes) to improve the chances of the richness of the data being retained by others.

\section{Preregistration and Registered Reports}

Preregistration and registered reports are key OS practices. With preregistration, one writes up information including study rationale, hypotheses, design and analysis plan into a document, and uploads it to an online repository. Registered reports take the preregistration process further, involving the peer-review of the preregistration document through a publishing outlet, just as in the review process of a fully complete research manuscript. Once the preregistration plan has been accepted, the study has been accepted by the publisher in principle, irrespective of its outcomes, providing the authors have stuck to their plan or describe any deviations in detail (Field et al., 2020).

Haven and van Grootel (2019) outline three key arguments one might have against using preregistration for qualitative study planning: 1) the goal of qualitative research (to generate rather than test theories) makes it unsuitable for preregistration/registered reports,

2) the flexibility required for many qualitative research approaches, and 3) subjectivity of qualitative research, both of which interfere with preregistering study plans. 
Haven and van Grootel counter these arguments. They suggest that opening a study's plans to the scrutiny of the scientific community is valuable despite qualitative research's lack of dependence on hypotheses. Flexibility is no issue with preregistered qualitative research either, they say. Pre-specifying a qualitative study design remains valuable and a valid act of transparency, even if it is the case that the design changes during the course of the study. Finally, Haven and van Grootel tackle the issue of subjectivity and preregistration, writing that subjectivity actually increases the value of preregistration. If a researcher chooses to preregister details about their traditional and theoretical interpretation lens, describes positionality, and reflects on their personal values before data collection and analysis, consumers of the eventual published study can use this contextual information to frame the findings and interpretations in question.

We agree that publishing one's research plans in advance can be useful in qualitative research, even if it is not strictly called preregistration. It can be used to invite commentary and suggestions from peers, for example, from which the study can benefit. This is also advanced as one of the advantages of registered reports: peer review in advance of the study can prevent problems that are difficult or impossible to repair once the data has been 'collected'. Keeping track of the vicissitudes of the research process can also be helpful and informative, both for the researchers themselves as for their peers. It can be interesting to know which avenues were abandoned during the course of the study, and why. Preregistration in any field can serve as a reminder to the authors themselves of what they intend to do, and to communicate to others what is intended.

\section{Other Open Science Practices}

Open Access and Open Peer Review. Open access and open peer review confer benefits to science, as well as society, as discussed previously, and are highly accessible for qualitative research fields. When authors do not have access to funding to publish in gold open access journals (where an author or institution pays article processing fees), green open access is still possible. Green open access refers to when an article has been made available on an online repository (e.g., Open Science Framework or arXiv). While it is often against copyright to upload the journal's copy edited version of the article, most journals do not expressly prohibit the posting of the final draft version independently online.

For qualitative researchers who have access to funding for gold open access, there are countless qualitative research journals indexed, including well-known journals such as the International Journal of Qualitative Methods, Forum: Qualitative Social Research, Social Science $\&$ Medicine and Cultural Anthropology. These individual journals offer Gold OA. Open access book publishers exist too. For example, Mattering Press is a UK charity which was established and is maintained by science and technology researchers who volunteer their time to provide online open access books for free for authors who are unable to pay fees.

Open peer review means that peer review documentation is made open alongside the article. Although the adoption of open peer review is a little behind open access, there are still several high-profile journals which use open peer review and publish qualitative research. These include journals under the PLOS publishing banner, the British Medical Journal, and Royal Society Open Science.

Citizen Science. Citizen science refers to when research is conceived of, designed and carried out with participation from the public. Such a partnership can be mutually 
advantageous: the research community's capacity is augmented by citizens participating in the research process, while the public's understanding of science and its output is enhanced (for an excellent example of citizen science in practice, see Okop et al., 2021). The quality and appropriateness of the research is enhanced too, as input from citizens ensures they are represented in what is studied, and how.

Citizen science can be seen as a powerful way to further reinforce the already close connection between qualitative researchers and their participants. Citizen science is, naturally, not suitable for all qualitative research projects, however in cases where the research findings are likely to benefit a community or group of people, engaging in citizen science is a worthwhile investment in OS for the qualitative researcher.

\section{Supporting the Adoption of Open Science}

Despite guides on the use of sensitive data repositories such as that of Manneheimer and colleagues, or the articles on OS practice adoption by Cruwell and colleagues (2019) and Kathawalla, Silverstein and Syed (2021), navigating the issue of sensitive data sharing is still a difficult task. Researchers should be supported in engaging with OS. This goes for researchers in all disciplines, and for all aspects of OS engagement.

\section{Support}

First, researchers need support to adopt OS in general. Good, slow science can be time- and labor-intensive. There exist such a number and variety of OS initiatives and events available for interested researchers to engage with, that entering into OS practice can be overwhelming (Kathawalla et al., 2021; Whitaker \& Guest, 2020; Robson et al., n.d.; Crüwell et al., 2019), and especially so for those who are still learning how to integrate OS into their research practice. If, as Corti (2000) suggested, qualitative researchers are indeed not used to the expectation of sharing their data, care should be taken to recognize the legitimacy of their concerns. Respect for the epistemic traditions of qualitative research should be shown by others who are more immersed in the OS movement.

Protocols and infrastructure available for data sharing should evolve to provide support for qualitative researchers who are motivated to share their data. Academic institutions and funding agencies should support the ethical obligations qualitative researchers have to protecting their participants (Chauvette et al., 2019). The good news is that the literature is starting to supply researchers with information on how to prepare their qualitative data for sharing. For example, McGrath and Nilsonne (2018) listed five measures for ethical qualitative data sharing. These measures include: a) sharing with 'stepped access', which is similar to the 'controlled' access defined by Jones and colleagues (2018) but with a structured protocol set in place to handle and review data access requests from researchers, and b) member checking, wherein participants are explicitly involved in checking the data before it is shared. This measure places the locus of control with participants, and allows for a "fully autonomous and informed decision" (p. 7) to share the data.

\section{Mentoring and Education}

Another element that can greatly facilitate qualitative researchers adopt OS, particularly with data sharing, is mentoring. For most practical scenarios, data sharing requires 
specialized skills which the average individual researcher (qualitative or quantitative) may not possess. This is an opportunity for institutional libraries. If each research institute had librarians or data managers on staff trained specifically to assist qualitative researchers prepare their data for sharing and safe storage, it would be easier for people to engage in data sharing practices. Jones and her colleagues (2018) suggest that dedicated professional data management staff might assist in guiding the researcher through decisions involving the required level of data processing, which varies as a function of the nature and context of the data and encompassing study.

Education and research are inherently entwined, say de Knecht, van der Meer, Brinkman, Kluijtmans, and Miedema (2021) in their article on connecting education and OS. They point out that education has a central role to play in shaping what they call the 'open academic self', and emphasize that in order for science to become more open and inclusive in future, educational reform is just as important as scientific reform. If 'OS education' were prioritized in universities, it would be a prime opportunity to educate students on the challenges of open qualitative as well as those of open quantitative research.

\section{Conclusion}

Most OS practices are straightforward and effective when applied to qualitative research. Qualitative scientists can open their research pipeline, and write manuscripts transparently and describe methods and analysis strategies with enough detail that others can follow what was done. They can preregister research plans, even when some details are unknown at the outset, and publish in journals and books which provide open access and open peer review.

In many cases too, data sharing is possible, and even straightforward. Though, as we discussed above, qualitative researchers must contend with two serious and legitimate issues when considering sharing their data. They are generally concerned about ethical and/or legal breaches when it comes to sharing their data, and fear that their data will be rendered one-dimensional and potentially useless should it be decontextualized. A cost-benefit tradeoff should be considered for every case involving the opening up of qualitative data, as the limitations to sharing rich and sensitive data are balanced by a slew of positive benefits to participants, the individual researcher, the scientific community and the public.

\section{Open Science as a Buffet}

Before closing on our final thoughts, we would like to consider whether or not qualitative researchers have to share their data to be reasonably considered an OS practitioner by the scientific community? Even if there are reasonable ways and means for doing so, the answer is 'probably not'. Bergmann, in a talk in 2018, called OS a buffet, and suggested that people adopt OS at a rate and to a degree that suits them, rather than doing it all, and ending up exhausted.

This, to us, implies that we can (and should) take each qualitative study as a single case, rather than using a one-size-fits-all approach to expectations about opening up qualitative research data (which Lorenz and Holland recommend against, anyway; Lorenz \& Holland, 2020). Whitaker and Guest (2020) put it elegantly, when they said that "... the recommendations that work in one context may not be applicable in another. They will 
need to be adapted according to local needs in a framework that is sensitive to community specific power structures." Some qualitative studies produce data which are much more suitable to be anonymized and shared than other studies, and some ethical boards will be much more open to the idea of building open data policies into ethical approval of qualitative research than will others. In some cases, the cost-benefit balance may not clearly favor sharing. In such cases, authors can choose to share highly processed data, or choose to share data under restrictions to a repository or in a controlled manner (for these categories, see Jones et al., 2018). They could choose not to share their data for that particular project, and instead invest time in contributing to or engaging in other OS practices.

Concerns about data sharing should not act to deter qualitative researchers from adopting OS more broadly. We suggest, for those who doubt that sharing data would yield a net positive, to avoid throwing the baby out with the bathwater. Take of OS what works best, and what is realistic for your methods and data, and consider what these requirements and limitations are on a case-by-case basis. We encourage qualitative researchers who want to engage with the spirit of OS to choose against sharing their data if they believe it is not ethically reasonable, and engage with the myriad other OS practices in existence which are more compatible with their line of research. This principle goes for more than just data sharing. As with practitioners of quantitative research, one does not have to engage in all OS practices to produce transparent research whose methods and findings stand up to scrutiny.

Whitaker and Guest (2020) stretch Bergmann's metaphor. They prompt readers to imagine the OS buffet as a pot-luck, a meal where different researchers bring different skills, approaches, questions - dishes — to the table of OS. Members can contribute in the way in which they're able, and OS as a community is free from the limitations of homogeneity, and benefit from greater diversity. They advance the point that the essence of OS is the same as the motivation for conducting research in the first place, that is, to learn from and educate others.

In this same vein, we suggest that OS has much to learn from the approaches of qualitative science. As others have previously argued (Field \& Derksen, 2021), the current era of crisis in science is marked by a near obsessive focus on objectivity and control. At first glance, one may be inclined to conclude that this emphasis - eliminating human bias, and maximizing reproducibility - amplifies the voice of quantitative research in the OS discussion, potentially attenuating that of the qualitative. While replication is not all that relevant to qualitative research, which aims to determine the how and why questions behind human phenomena through the collection and interpretation of complex and rich data, reproducibility is still very much on the agenda. As Dienlin and colleagues (2021) say, qualitative science has modest but certain aims with regard to transparency, generalizability and validity. Lorenz and Holland share the perspective that qualitative researchers are perhaps even more attuned to the goals of OS than their quantitative counterparts, as they routinely confront issues of personal bias and justice and inequality in translating and sharing research findings. This is achieved, in part, through reflexivity.

Reflexivity as a route toward open science. Reflexivity is the term given to a practice popular in sociological and anthropological fields. It describes a state of constant self-awareness the researcher actively engages in, as they move through the research process. Ideally, according to Finlay (2002), it should begin the moment the research idea comes into 
being in the minds of the researchers, and should be an active operation throughout each stage in the research process (Hammersley \& Atkinson, 1995). The practice of reflexivity is used to help the researcher gain awareness of their doxa - that is, often taken for granted, or at least tacit, values and beliefs - and consider how these doxa interact with knowledge (co-)production (Field \& Derksen, 2021). Bourdieu (1972) wrote that in challenging our doxa, we have the potential to bring the "undiscussed into discussion, and the unformulated into formulation" (p. 168).

We would like to point out too, that reflexivity is not just something the researcher engages in. It is something which is shared in the research report (positionality statements are an example of this, but written reflections can take other explicit forms too, see e.g., the opening reflection in Field \& Derksen, 2021). In other words: reflections are frequently made public and transparent, a kind of openness which is very much in line with OS philosophy.

Awareness and acknowledgment of ones identity, as achieved by reflexivity, is made concrete and is linked to a specific research context through a positionality statement. Positionality statements are how a researcher can delineate their position in relation to the study, with the implication that that position may influence aspects of the study. They are a valuable tool to help reduce "bias and partisanship" (p. 4; Holmes, 2020). They are a means by which researchers state concretely their 'lenses' - perspectives, beliefs and attributes (such as their gender, sex, age, social class and ethnicity) which influence their view of the research process and impact upon the choices they make as they conduct their research. Positionality is related to reflexivity very closely, in that reflexivity informs positionality (Holmes, 2020). Reflexivity and positionality are examples of 'dishes' which qualitative researchers can contribute to the OS buffet. We argue that OS would benefit from its practitioners exercising the explicit interrogation of their biases and positions.

The buffet metaphor, however, should not obscure the fact that introducing OS practices in qualitative research, even if it is the result of individual choice (from a buffet) rather than imposed from above (as, say, a fixed menu) has consequences for qualitative research in general. Transparency is performative: it does not merely make things visible, but it is liable to change what there is to see in the first place. This is particularly pertinent where OS practices function to facilitate inspection and surveillance. The distrust this implies may be warranted, and suspicion is already an element of qualitative research, but the kind of scrutiny that is introduced influences the character of the field.

\section{Final Thoughts}

Opening up research in any discipline comes with growing pains and inertia for all spheres of scientific inquiry. OS can be difficult, time-consuming and daunting, and cannot be reasonably applied in the same way to qualitative disciplines as for quantitative disciplines. That said, qualitative research has a natural affinity for openness and transparent practice, owing to its reliance on methods like reflexivity. Qualitative OS is open science, just perhaps with a lighter emphasis on data sharing, and a heavier emphasis on understanding researcher bias and positionality. As we have argued, most OS practices are achievable and should be the goal for qualitative researchers. 


\section{References}

Borgman, C. L., Wofford, M. F., Golshan, M. S., \& Darch, P. T. (2021). Collaborative qualitative research at scale: Reflections on 20 years of acquiring global data and making data global. Journal of the Association for Information Science and Technology, 72(6), 667-682.

Bourdieu, P. (1972). Outline of a theory of practice. translated by richard nice. Cambridge studies in social anthropology, 16.

Chauvette, A., Schick-Makaroff, K., \& Molzahn, A. E. (2019). Open data in qualitative research. International Journal of Qualitative Methods, 18, 1609406918823863.

Coltart, C., Henwood, K., \& Shirani, F. (2013). Qualitative secondary analysis in austere times: Ethical, professional and methodological considerations. Historical Social Research/Historische Sozialforschung, 271-292.

Corti, L. (2000). Progress and problems of preserving and providing access to qualitative data for social research - the international picture of an emerging culture. In Forum qualitative sozialforschung/forum: Qualitative social research (Vol. 1).

Corti, L., \& Fielding, N. (2016). Opportunities from the digital revolution: Implications for researching, publishing, and consuming qualitative research. Sage open, 6(4), 2158244016678912 .

Crüwell, S., van Doorn, J., Etz, A., Makel, M. C., Moshontz, H., Niebaum, J. C., ... Schulte-Mecklenbeck, M. (2019). Seven easy steps to open science. Zeitschrift für Psychologie.

de Knecht, S., van der Meer, M., Brinkman, L., Kluijtmans, M., \& Miedema, F. (2021, August). Reshaping the Academic Self: Connecting Education 83 Open Science. Zenodo. Retrieved from https://doi.org/10.5281/zenodo.5345573 doi: 10.5281/ zenodo. 5345573

Dienlin, T., Johannes, N., Bowman, N. D., Masur, P. K., Engesser, S., Kümpel, A. S., ... others (2021). An agenda for open science in communication. Journal of Communication, 71(1), 1-26.

Field, S. M., \& Derksen, M. (2021). Experimenter as automaton; experimenter as human: exploring the position of the researcher in scientific research. European Journal for Philosophy of Science, 11(1), 1-21.

Field, S. M., Wagenmakers, E.-J., Kiers, H. A., Hoekstra, R., Ernst, A. F., \& van Ravenzwaaij, D. (2020). The effect of preregistration on trust in empirical research findings: Results of a registered report. Royal Society Open Science, 7, 181351.

Finlay, L. (2002). "outing" the researcher: The provenance, process, and practice of reflexivity. Qualitative health research, 12(4), 531-545.

Hammersley, M., \& Atkinson, P. (1995). Recording and organizing data. Ethnography: Principles in Practice. London: Routledge, 175-204.

Holmes, A. G. D. (2020). Researcher positionality-a consideration of its influence and place in qualitative research-a new researcher guide. Shanlax International Journal of Education, 8(4), 1-10.

Jackson, R. L., Drummond, D. K., \& Camara, S. (2007). What is qualitative research? Qualitative research reports in communication, 8(1), 21-28.

Jones, K., Alexander, S. M., Bennett, N., Bishop, L., Budden, A., Cox, M., ... others 
(2018). Qualitative data sharing and re-use for socio-environmental systems research: A synthesis of opportunities, challenges, resources and approaches (Tech. Rep.).

Kathawalla, U.-K., Silverstein, P., \& Syed, M. (2021). Easing into open science: A guide for graduate students and their advisors. Collabra: Psychology, 7(1).

Kowalczuk, M. K., Dudbridge, F., Nanda, S., Harriman, S. L., \& Moylan, E. C. (2013). A comparison of the quality of reviewer reports from author-suggested reviewers and editor-suggested reviewers in journals operating on open or closed peer review models. F1000Research, 4 .

Lezaun, J., \& Montgomery, C. M. (2015). The pharmaceutical commons: Sharing and exclusion in global health drug development. Science, Technology, $\&$ Human Values, $40(1), 3-29$.

L. Haven, T., \& Van Grootel, D. L. (2019). Preregistering qualitative research. Accountability in Research, 26 (3), 229-244.

Lincoln, Y. S., \& Guba, E. G. (1985). Naturalistic inquiry. sage.

Lorenz, T. K., \& Holland, K. J. (2020). Response to sakaluk (2020): Let's get serious about including qualitative researchers in the open science conversation. Archives of Sexual Behavior, 49(8), 2761-2763.

Mannheimer, S., Pienta, A., Kirilova, D., Elman, C., \& Wutich, A. (2019). Qualitative data sharing: Data repositories and academic libraries as key partners in addressing challenges. American Behavioral Scientist, 63(5), 643-664.

McBee, M. T., Makel, M. C., Peters, S. J., \& Matthews, M. S. (2018). A call for open science in giftedness research. Gifted Child Quarterly, 62(4), 374-388.

McGrath, C., \& Nilsonne, G. (2018). Data sharing in qualitative research: Opportunities and concerns. MedEdPublish, $\%$.

Miller, K. (2015). Dear critics: Addressing concerns and justifying the benefits of photography as a research method. In Forum qualitative sozialforschung/forum: Qualitative social research (Vol. 16, p. 17).

Okop, K. J., Murphy, K., Lambert, E. V., Kedir, K., Getachew, H., Howe, R., ... Rulisa, S. e. a. (2021). Community-driven citizen science approach to explore cardiovascular disease risk perception, and develop prevention advocacy strategies in sub-saharan africa: a programme protocol. Research involvement and engagement, 7(1), 1-14.

Pain, H. (2012). A literature review to evaluate the choice and use of visual methods. International Journal of Qualitative Methods, 11(4), 303-319.

Pownall, M., Talbot, C. V., Henschel, A., Lautarescu, A., Lloyd, K. E., Hartmann, H., ... Siegal, J. (2021). Navigating open science as early career feminist researchers. Psychology of Women Quarterly.

Pratt, M. G., Kaplan, S., \& Whittington, R. (2020). Editorial essay: The tumult over transparency: Decoupling transparency from replication in establishing trustworthy qualitative research. Administrative Science Quarterly, 65(1), 1-19.

Roberts, K., Dowell, A., \& Nie, J.-B. (2019). Attempting rigour and replicability in thematic analysis of qualitative research data; a case study of codebook development. $B M C$ medical research methodology, 19(1), 1-8.

Robson, S. G., Baum, M. A., Beaudry, J. L., Beitner, J., Brohmer, H., Chin, J., ... others (n.d.). Nudging open science.

Sarafoglou, A., Hoogeveen, S., Matzke, D., \& Wagenmakers, E.-J. (2020). Teaching good 
research practices: Protocol of a research master course. Psychology Learning \& Teaching, 19(1), 46-59.

Spellman, B. A., Gilbert, E. A., \& Corker, K. S. (2018). Open science. Stevens' Handbook of Experimental Psychology and Cognitive Neuroscience, 5, 1-47.

Stake, R. E. (1995). The art of case study research. sage.

Standen, E. (2019). Open science, pre-registration and striving for better research practices. Psychological Science Agenda.

Vaismoradi, M., Turunen, H., \& Bondas, T. (2013). Content analysis and thematic analysis: Implications for conducting a qualitative descriptive study. Nursing 8 health sciences, $15(3), 398-405$.

Vicente-Saez, R., \& Martinez-Fuentes, C. (2018). Open science now: A systematic literature review for an integrated definition. Journal of business research, 88, 428-436.

Walsh, E., Rooney, M., Appleby, L., \& Wilkinson, G. (2000). Open peer review: a randomised controlled trial. The British Journal of Psychiatry, 176(1), 47-51.

Whitaker, K., \& Guest, O. (2020). \# bropenscience is broken science: Kirstie whitaker and olivia guest ask how open 'open science'really is. The Psychologist, 33, 34-37. 